\title{
Obesity and spinal cord injury: an observational study
}

\author{
Jeff Blackmer and Shawn Marshall \\ Department of Rehabilitation Medicine, University of Saskatchewan, Saskatoon, SK, Canada
}

\begin{abstract}
Obesity as an independent factor influencing the eventual rehabilitation outcome of spinal cord injured individuals has not been examined. This paper is an observational study of two patients with complete tetraplegia secondary to spinal cord injury. Both patients encountered several problems specifically related to their obesity which interfered with the rehabilitation process, and both were far below the expected functional outcome level for a $\mathrm{C} 7$ tetraplegic individual at the time of their discharge. Both patients were discharged to facilities providing the highest level of care available in the province.
\end{abstract}

Keywords: tetraplegia; obesity; function

\section{Introduction}

Several factors have been identified which can have an influence on the functional outcome for spinal cord injured patients. These factors include age, pain, spasticity, the centre where rehabilitation takes place, the degree of neurological completeness of the lesion, the lesion level and motor function at the time of injury. ${ }^{1-7}$ Obesity is another factor which could conceivably alter functional outcome but has not been examined in detail. Obesity can be defined as a body mass index (body weight in kilograms divided by height in meters squared) or BMI in the 85th percentile or higher. The 85 th percentile is 27.8 for males and 27.3 for females. The Surgeon General's 1988 Report on obesity notes that it increases the risk for other impairments such as premature death, diabetes, hypertension, and atherosclerosis, however the issue of how obesity affects function or in essence creates disability is not addressed. ${ }^{8}$ It would seem logical that an obese patient with spinal cord injury might encounter unique problems during their rehabilitation and post-discharge which could be directly or indirectly attributed to their excess weight and that obesity itself might interfere with their eventual functional outcome.

In this paper we will discuss two patients who underwent inpatient rehabilitation for $\mathrm{C} 7$ complete tetraplegia. We will review the problems and difficulties encountered with their rehabilitation as a direct or indirect result of their weight status. These problems included medical complications, special nursing implications and special equipment needs. We will also compare their eventual functional outcome to the predicted functional outcome for $\mathrm{C} 7$ tetraplegic patients.

Correspondence: J Blackmer

\section{Patient A}

Patient A was a 35 year old female who weighed $181.8 \mathrm{~kg}$ and had a height of 1.68 metres at the time of her admission, equating to a BMI of 64.5. Premorbidly, she had a functional level normal for a person her age. She was first seen in the emergency department with a complaint of being unable to walk. The patient had a 3 month history of progressive bilateral limb numbness and weakness followed by acute onset of complete tetraplegia secondary to a centrally herniated intervertebral disc at the $\mathrm{C} 6-\mathrm{C} 7$ level. She underwent emergency $\mathrm{C} 6-\mathrm{C} 7$ discectomy to decompress the cord but post operatively she remained with $\mathrm{C} 7$ complete tetraplegia. Her total acute care time was 33 days and her rehabilitation stay was 99 days. Her past medical history included asthma, Type II diabetes mellitus, morbid obesity and a history of smoking.

\section{Patient B}

Patient B was a 46 year old gentleman with C7 complete tetraplegia who weighed approximately 113.9 $\mathrm{kg}$ and was 1.73 metres tall on his admission resulting in a BMI of 38.1. Prior to his admission to hospital he also had a normal functional level for a person his age. He suffered an assault, resulting in a traumatic fracture through the $\mathrm{C} 6-\mathrm{C}$ disc space with complete disruption of all three columns. He subsequently had a spinal operation, with Luque rod instrumentation extending from $\mathrm{C} 2$ to $\mathrm{T} 3$. His acute care stay was 25 days and his rehabilitation stay was 130 days. His past medical history included chronic, morbid obesity for which gastric bypass surgery had been unsuccessfully tried in 1979, ankylosing spondylitis, a long history of smoking and a previous history of alcohol abuse. He was a widower who lived alone. 


\section{Discussion}

Obesity has not been previously examined as an independent factor influencing the expected functional outcome of spinal cord injured patients. Both patients reviewed in this paper were defined as obese according to the Surgeon General's criteria and each were relatively young and healthy, although Patient B had a past history of ankylosing spondylitis.

Studies of psychological distress following spinal cord injury have implicated several factors including level of distress at the time of admission, neurological completeness of the spinal cord injury, type of rehabilitation insurance, gender, marital status and age. ${ }^{15,16}$ Obesity was not examined as a possible factor although it has been shown to be associated with depression in the non-spinal cord injured population, and therefore may contribute to anxiety in spinal cord injured patients as well. ${ }^{17,18}$ Both patients in this study encountered emotional and psychological hurdles, including depression, anxiety and claustrophobia, and these difficulties also required extra nursing attention and care.

Both patients developed medical complications during their stay which were felt to be due to at least in part to their obesity. Patient A developed a pulmonary embolism and subsequent respiratory compromise. It could be argued that due to Patient A's large size, a deep venous thrombosis would be less likely to be identified clinically and in this instance more aggressive monitoring or prophylaxis may have been warranted. A spinal cord injured patient generally breathes more comfortably and efficiently while lying on his/her back, as the abdominal contents will help displace the diaphragm for respiratory excursion. ${ }^{11}$ However, Patient A did have more difficulty breathing while lying on her back due to the excessive weight of her abdominal wall making diaphragmatic excursion more difficult. This may have been a significant contributor to her respiratory compromise.

Both patients developed what appeared to be ulnar nerve neuropathies with entrapment at the level of the elbow. In one study of compressive mononeuropathies in paraplegic patients, $67 \%$ of patients had evidence of at least one compressive mononeuropathy according to electrodiagnostic criteria. ${ }^{19}$ This included $10 \%$ of patients with an ulnar nerve conduction block across the elbow. It ws suggested that compressive mononeuropathies of the ulnar nerve at the elbow may be a function of repetitive trauma in the course of propelling a wheelchair, and presumably this risk would increase with a heavier limb. Another factor to be considered would be direct compression of the nerve attributable to resting the forearms on the wheelchair arm rests.

The excess weight of the patients caused several special nursing considerations. Both initially required up to a three person assist for transfers, bed turning and catheterization. A considerable amount of time was required of nursing staff to transfer them into bed in order to perform catheterization which could not be done sitting up because of their size. All these factors placed an excess amount of burden on the staff in terms of time required for care. There was also the physical burden of turning and assisting an obese patient for self care. Of even more importance than the level of effort and assistance required of nursing staff is the significant risk of injury to these nurses when working with morbidly obese, heavily dependent patients. Two documented back injuries resulted from turning Patient A. Injury is a common concern of nursing staff who care for obese patients and has been documented elsewhere. 9,10

Both patients in our study were found to have functional outcomes which were significantly poorer than what would be expected for a patient with a spinal cord injury at the C7 level. ${ }^{11-14}$ In the area of self care, both patients were reasonably near their expected outcomes in the areas of eating and grooming. However, in the more physically demanding activities of bathing, dressing and toileting, they remained extremely dependent; Patient B was completely so.

Neither was able to gain any independence in the areas of bowel and bladder management, although most tetraplegics at the $\mathrm{C} 7$ level are able to do so. Their abdominal obesity made it impossible for them to independently gain access for self-catheterization. By the same token, they would be unable to selfadminister bowel care due to their restricted reach, once again secondary to the physical barrier of obesity.

Both patients were expected to become fairly independent for mobility and locomotion. However, although both were able to manoeuver a wheelchair to some degree, neither attained full independence in these areas. Because of their obesity, neither was able to support their body weight with their arms and they were therefore totally dependent for transfers. Patient B required a tilt-in-space electric wheelchair since he was unable to provide pressure relief. Patient A was still able to propel a manual ultralight wheelchair.

Although it is very difficult to quantify precisely, it is obvious that there would be a greatly increased cost of care involved with these patients. Their medical problems, such as the deep vein thrombosis and pulmonary embolism of Patient A, would increase the cost significantly. Patient A required an expensive stay in the ICU due to this complication, in addition to numerous consultations and medications. The burden placed on the nursing staff meant that more nurses had to be available to assist with the care of these patients, also at an extra cost to the system.

The average length of stay for a traumatic spinal cord injured patient at our facility during the year when these patients were admitted was 62 days. The cost to stay at our rehabilitation facility is presently $\$ 622$ (Canadian dollars) per day. The length of stay 
for Patient A was 99 days, which repesents an extra cost of $\$ 16794$. For Patient B, whose length of stay was 130 days, the extra cost would be $\$ 39808$.

Finally, and most signficantly, both patients required placement at facilities with the highest level of care available in the province, defined as greater than $2 \mathrm{~h}$ of nursing care daily. Becoming independent in society is generally the ultimate goal of someone with C7 level tetraplegia but neither patient was able to obtain this, largely due to their obesity. This represents a significant ongoing cost to the system.

\section{Conclusion}

Obesity can be considered to be a factor which can contribute to impaired functional outcome in spinal cord injured patients compared to no obesity in such patients with a similar neurological injury level. This is significant since obesity generally does not necessarily contribute to disability in others without a neurological deficit. There is an increased risk of complications for the patients themselves, as well as an increased burden placed on the nursing staff which is directly related to the excess weight of the patient.

\section{References}

1 Woolsey RM. Rehabilitation outcome following spinal cord injury. Arch Neurol 1985; 42: 116-119.

2 Welch RD et al. Functional independence in quadriplegia: critical levels. Arch Phys Med Rehabil 1992; 67: 235-240.

3 DeVivo MJ, Stover SL, Black KJ. Prognostic factors for 12-year survival after spinal cord injury. Arch Phys Med Rehabil 1992; 73: $156-162$.

4 Heinemann AW, Yarkony GM, Roth EJ. Functional outcome following spinal cord injury. A comparison of specialized spinal cord injury center vs. general hospital short term care. Arch Neurol 1989; 46: $1098-1102$.
5 DeVivo MJ et al. The influence of age at the time of spinal cord injury on rehabilitation outcome. Arch Neurol 1990; 47: 687691.

6 Penrod LE, Hedge SK, Ditunno JF Jr. Age effect on prognosis for functional recovery in acute, traumatic central cord syndrome. Arch Phys Med Rehabil 1990; 71: 963 -968.

7 Lazar RB et al. Prediction of functional outcome by motor capability after spinal cord injury. Arch Phys Med Rehabil 1989; 70: $818-822$

8 The Surgeon General's Report on Nutrition and Health. US Department of Health and Human Services Public Health Service Publication 88-50210, 1988.

9 Brentin L, Sieh A. Caring for the morbidly obese. Amer $J$ of Nursing 1991; 91: 40-43.

10 Hudelson E. Points to remember when caring for an obese patient. Nursing 1992; 22: $62-63$.

11 DeLisa JA, Gans BM. Rehabilitation Medicine: Principles and Practice, 2nd edition. Philadelphia: JB Lippincott Company, 1993.

12 Long C, Lawton EB. Functional significance of spinal cord lesion level. Arh Phys Med Rehabil 1955; 36: 249-255.

13 Symington MB, Mackay DE. A study of functional independence in the quadriplegic patient. Arch Phys Med Rehabil 1966; 47: $378-392$.

14 Guide for the uniform data set for medical rehabilitation (Adult FIM). Version 4.0. UB Foundation Activities, Inc. 1993.

15 Tate DG Maynard F, Forchheimer M. Predictors of psychological distress one year after spinal cord injury. Am J Phys Med Rehabil 1993; 72: $272-275$.

16 Tate DG et al. Predicting depression and psychological distress in persons with spinal cord injury based on indicators of handicap. Am J Phys Med Rehabil 1994; 73: 175-183.

17 Schwartz F. Obesity in adult females: the relationship among personality characteristics, dieting and weight. AAOH Journal 1993; 41: $504-509$

18 Stewart AL, Brook RH. Effects of being overweight. Am J Public Health 1983; 7: $171-178$.

19 Davidoff G, Werner R, Waring W. Compressive mononeuropathies of the upper extremity in chronic paraplegia. Paraplegia 1991; 29: $17-24$ 